\title{
The effect of progressive muscle relaxation training on chemotherapy-induced nausea, vomiting and anxiety in Egyptian breast cancer women: A randomized controlled trial
}

\author{
Hanan Mohamed Soliman*1, Ahmed Eltantawy ${ }^{2}$, Rania El-Kurdy ${ }^{3}$ \\ ${ }^{1}$ Faculty of Nursing, Medical-Surgical Nursing Department, Mansoura University, Egypt \\ ${ }^{2}$ Medical Oncology Department, Mansoura University, Egypt \\ ${ }^{3}$ Woman's Health and Midwifery Nursing Department, Faculty of Nursing, Mansoura University, Egypt
}

Received: August 5, 2021

DOI: $10.5430 /$ jnep.v12n $4 \mathrm{p} 1$
Accepted: November 7, $2021 \quad$ Online Published: November 15, 2021

URL: https://doi.org/10.5430/jnep.v12n4p1

\begin{abstract}
Background and objective: This study was conducted to examine the effect of progressive muscle relaxation training (PMRT) in combination with antiemetic drugs on chemotherapy-associated nausea, vomiting and anxiety in breast cancer women receiving chemotherapy.

Methods: A randomized controlled trial design was conducted on 74 patients divided into control $(\mathrm{n}=37)$ and PMRT group $(\mathrm{n}=$ 37). The study was conducted at Oncology Center affiliated to Mansoura University, Egypt. The intervention included daily PMRT practice for seven days two hours before chemotherapy. The patients exercised in the oncology unit with the researcher, and individually applied the exercises at home. The data was collected using Rhodes index of nausea-vomiting and retching used daily after chemotherapy for seven days and Zung self-rating anxiety scale used before chemotherapy and at the seventh and 14th days after chemotherapy.

Results: A significant decrease in the frequency, severity, and duration of vomiting, nausea, retching and anxiety in PMRT group than in the control group $(p<.0001)$.

Conclusions: Progressive muscle relaxation combined with antiemetics was effective in reducing vomiting, nausea, and retching, as well as improving anxiety level induced by chemotherapy.
\end{abstract}

Key Words: Anxiety, Breast cancer, Chemotherapy, Nausea-vomiting, Progressive muscle relaxation

\section{INTRODUCTION}

Breast cancer (BC) is the most common cause of cancer deaths among women worldwide. Incidence of $\mathrm{BC}$ is high in most developed countries while rates are lower in developing countries. More than 180,000 American women are diagnosed with breast cancer annually. ${ }^{[1]}$ In Egypt, BC is the second-leading cause of mortality in women, it accounts for $33 \%$ of female cancer cases and more than 22,000 new cases diagnosed each year. Cancer incidence rate in general 157.0 per 100,000 female Egyptian with the highest rate to BC, by the year 2050, cancer rates are expected to be three-fold. ${ }^{[2]}$ There are various methods of cancer treatment which include

\footnotetext{
*Correspondence: Hanan Mohamed Soliman; Email: hanansoliman697@ymail.com; Address: Faculty of Nursing, Medical-Surgical Nursing Department, Mansoura University, Egypt. 
chemotherapeutic agents, radiation therapy, immunotherapy, surgical managements etc. Nevertheless, these treatment modalities cause many distress symptoms that negatively affects the quality of life of patients. There are many distressing effects of chemotherapy used in breast cancer, including: anorexia, nausea-vomiting, anxiety, insomnia, dyspnea, and stomatitis. Chemotherapy can be used as a single treatment or combined with other treatment modalities. ${ }^{[3]}$

Vomiting and nausea are the most common distressing symptoms, which may last between 24 and 72 hours after chemotherapy and gradually resolve over the next several days. ${ }^{[4]}$ Up to $66 \%-91 \%$ of cancer patients receiving chemotherapeutic agents experienced nausea and vomiting. Direct effects of Prolonged chemotherapy-induced nausea and vomiting (CINV) can cause anxiety, and Malnutrition. Some patients reported that complications from cancer treatment are worse than the cancer itself. Additionally, some patients discontinue chemotherapeutic course, which reduces the hope for recovery. Hence, great efforts have been made to effectively prevent and alleviate Nausea and vomiting induced by chemotherapy. Recently, antiemetics are still the cornerstone in the treatment of nausea and vomiting caused by chemotherapy, while the preventive use of antiemetics is not applicable to all cancer patients who have been directed to receive chemotherapy. More importantly, anti-nausea and vomiting drugs reduce these complications, but they do not fully cure the problem. ${ }^{[5]}$ Thus, a combination of nonmedication treatments with medication-based treatments is recommended for reducing CINV. ${ }^{[6]}$

When shedding light on previous studies, mind-body practices such as progressive muscle relaxation (PMR), breathing exercise and meditation have been utilized recently to reduce anxiety, nausea-vomiting and fatigue in the individuals and improve their quality of life through voluntary stretching and relaxation of all the muscle of the body, from head to toe. ${ }^{[7,8]}$ The progressive muscle relaxation training (PMRT) is one of the most common non-pharmacological techniques to reduce the physical symptoms of chemotherapy, it has both a cognitive distraction and a relaxation element. ${ }^{[9-11]}$

Relaxation reduces physiological arousal and thus prevents the exacerbation of vomiting and nausea, as well as reduces feelings of stress and anxiety. Vomiting and nausea associated with chemotherapy may be controlled by relaxation that reduces gastrointestinal contractility or calming effect on vomiting center in the brain. ${ }^{[12]}$

\subsection{Significance of the study}

From researchers' clinical observation, it is noticed that CINV and anxiety are the most distressing side effects to deal with in clinical practice, that viewed as a challenge to patients, oncology nurses, and may hinder patients' adherence to drug. Proceeding from the fact that antiemetics alone are not effective in controlling CINV and anxiety, it is necessary to look for other methods either alternative or adjuvant to antiemetics. In this context, recent studies have reported that PMRT is one of the non-drug therapies that has shown a positive effect in controlling CINV in cancer patients. ${ }^{[13,14]}$ On the other hand, such techniques rarely used with Egyptian patients, in this regard, the current study aimed to examine the impact of PMRT along with antiemetics on nausea, vomiting, and anxiety induced by chemotherapy among BC women.

\subsection{Study aim}

The aim of this study was to examine the effect of PMRT in combination with antiemetic drug on CINV and anxiety in breast cancer women undergoing chemotherapy.

\subsection{Research hypotheses}

H1. The PMRT group would show significantly greater improvements in frequency, duration, and intensity of chemotherapy-induced nausea-vomiting and retching than the control group participants immediately and for a week post-chemotherapy.

H2. Women with breast cancer who receive PMRT will have significantly less anxiety than women in the control group after two weeks of chemotherapy.

\subsection{Theoretical framework}

Chan et al. multi-dimensional interventions in managing CINV

Basically, when the vomiting center is stimulated in the brain, vomiting and nausea occur. ${ }^{[15]}$ This happens through 4 paths as follows: (1) the chemoreceptor trigger zone, (2) the cerebral cortex and limbic system, (3) the vestibular system and (4) the afferent vagal and visceral nerves. Although the availability of antiemetics is increasing annually, these treatments alone are not effective against vomiting and nausea caused by multiple pathways. ${ }^{[15,16]}$ In this model, it has been suggested that a multi-dimensional action is more effective if more than one vomiting pathway is closed. It is a multi-dimensional intervention in managing CINV, serves as a pioneer framework which apply PMRT combined with patient education and antiemetics to achieve optimal goal of blocking all four emetic pathways. Relaxation blocks the cerebral cortex pathway, while patient education focuses on risks assessment and antiemetics blocks the other three pathways. Several studies have revealed that multi-dimensional interventions are effective in managing CINV in adults. ${ }^{[17,18]}$ PMRT is a method that uses a favorable and relaxing mental image to distract an individual from the side effects of chemotherapy. ${ }^{[19]}$ The 
study concluded that nurses can successfully demonstrate PMRT combined with patient education and antiemetics to control CINV among cancer patients. ${ }^{[20]}$

\section{RESEARCH METHODS}

\subsection{Design, setting, and participants}

A randomized controlled study design was conducted to investigate the effects of the PMRT on CINV in BC women. The study was conducted at Oncology Center of Mansoura University Hospitals, Dakahlia governorate, Egypt. Breast cancer women undergoing AC protocol of chemotherapy attend the previously mentioned setting constituted study subject. In this study, to ensure the homogeneity of the sample, one type of chemotherapy was selected as well as only one type of cancer (BC). Sample size was calculated by GPower software (version 3.1.9.7). The authors assume a medium effect size $(f=0.25)$ in a between-subjects design comparing the change over time in mean number of episodes of both vomiting and nausea (observed daily for one week). Accordingly, a total sample size of 74 participants from outpatient oncology clinics (37 in each arm) achieves a power of $80.15 \%$ to detect a medium effect size $(f=0.25)$ with an $\alpha$-level of $5 \%$, and correlation among repeated measures of 0.5 using repeated-measures ANOVA test (within-factors) with 7 repeated measurements (every day for 7 successive days).

Nausea and vomiting following chemotherapy for breast cancer occur in $60 \%-90 \%$ of cases and commonly begin within 1-2 hours and last for 6-12 hours with some patients experiencing vomiting and nausea for more than a day. In a previous study, the experimental subjects reported statistically significant lower attacks of vomiting and nausea than the control group, particularly in the first four days of administering chemotherapy $(p<.05){ }^{[18]}$

Inclusion criteria for the participants were (1) adult breast cancer women aged from 20 to 60 year, (2) did not expose to cytotoxic agents prior to the study (a first initial course), (3) Willing to participate in the study, and (4) able to read and write. Exclusion criteria were (1) subjects with CNS metastasis, a history of intestinal obstruction, sensitivity to dexamethasone, past history of end-stage malignancy and/or mental disorders. The treatment protocol for vomiting and nausea caused by chemotherapy used in the hospital when conducting the current study was Granisetron $0.01 \mathrm{mg} / \mathrm{kg}$ i.v. along with $10 \mathrm{mg}$ intra venous Dexamethasone. To be taken half an hour before a dose of chemotherapy administration. Also, 1-2 mg of Granisteron p.o. daily available for subsequent use.

Published by Sciedu Press

\subsection{Ethical considerations}

Ethical approval for the study was obtained from the Scientific Research Ethics Committee of Faculty of Nursing, Mansoura University. Informed consent was obtained from participants enrolled in the study after clarification the nature and aim of the study. The researcher emphasized that participation is voluntary. Anonymity, privacy, security, and confidentiality were maintained throughout the entire study. Participating patients were instructed that they had the right to withdraw from the study at any stage. The study was conducted in accordance with the institutional ethical standards, including the Helsinki Declaration.

\subsection{Procedure}

Once the necessary approval granted to proceed with the proposed study, subjects who met sampling criteria and accept to participate in the study were invited. The AC protocol for administering chemotherapy in the hospital, at the time of the present study, consisted of Adriamycin $60 \mathrm{mg} / \mathrm{m}^{2} \mathrm{I}$.V. (day 1) and Cyclophosphamide $600 \mathrm{mg} / \mathrm{m}^{2} \mathrm{I} . \mathrm{V}$ (day 1), repeated every three weeks for four consecutive cycles.

A pilot study was conducted before the main study on $10 \%$ of the total sample size ( 9 women) and minor modifications was done to the study design and data collection tools. As well as the time needed to complete the filling of the questionnaire was determined. The study was conducted over a period of 9 months from the beginning of February 2019 to the end of October 2020. Both PMRT and control groups were interviewed two hours before starting of chemotherapy and were provided with information about the study and were directed to a quiet room. All participants filled out questionnaires related to their level of anxiety (baseline data) before the start of chemotherapy and then on the seventh and fourteenth day after chemotherapy.

\subsection{Intervention}

In the first session, all patients in the intervention group received PMRT training from the researcher in a separate room. The researcher started by illustrating relaxation exercises, then the patients applied the exercises in the hospital, and daily for another five days at the patients' home, for a total of six sessions. In addition, they also received a brochure and a half-hour video demonstration of instructions on PMRT steps to implement at home. Patients were directed to perform the PMRT once a day and record the frequency and duration of exercises.

Furthermore, participants were received telephone call daily by the researcher to motivate them to exercise on the days they did not receive chemotherapy. The PMRT group were given antiemetics after a PMRT session. Vomiting and nau- 
sea were evaluated daily for a week. The control group was given antiemetics according to the hospital chemotherapy protocol half an hour before chemotherapy administration. In this context and daily for the first week of chemotherapy, the patients self-evaluated the severity, frequency, duration of vomiting and nausea using Rhodes index scale. After completing the study, the control group was directed on how to perform PMRT and they received brochures as well as an educational video.

\subsection{Application of PMRT}

PMRT involves deep breathing and progressive relaxation of eleven muscle groups (upper limbs, right and left calf, right and left foot, forehead, jaw, neck, back, shoulders, stomach, and thighs). The PMRT session starts by stretching - releasing muscle groups in the upper body first and then progressing to pulling - and releasing muscle groups in the lower body part. When applying PMRT, the women were trained to tighten the muscle group each according to their endurance without feeling pain with a deep breath, about 6-8 seconds, then relax for 10 seconds with a slow exhale and finally rested by taking a deep breath for 20-30 seconds before moving to the next muscle. This session took about 25 minutes to run.

\subsection{Data collection tools}

\subsubsection{Patient information and health relevant data form}

Include age, level of education, residence and occupation, marital status, and stage of disease. Current history including diagnosis, date of first chemotherapy dose, chemotherapy management protocol, current antiemetic drugs, etc.

\subsubsection{Rhodes index of nausea, vomiting and retching scale}

It consists of eight 5-point self-assessed items to measure subjective and objective vomiting, nausea, and retching. ${ }^{[21]}$ The INVR was a valid and reliable tool for assessing vomiting, nausea and retching ranged from 0.912 to $0.968 .{ }^{[22]}$ Scores for the individual items ranged from 0 to 4 , with higher scores indicating more nausea, vomiting, or retching. This scale was applied immediately after PMRT sessions and chemotherapy dose.

\subsubsection{Zung self-rating anxiety scale (SAS)}

It is a self-reported scale of 20 items that are rated on a fourpoint likert scale of "none or little time" (rating 1), "some time" (2), or "much of the time" (3) or "most of the time or all of the time" (4). The overall SAS score may range from 20 (free of anxiety) to 80 (severe anxiety). The higher the score, the higher the anxiety. ${ }^{[23]}$ Participants with a score of 36 were described by Zung as having clinically significant anxiety. The SAS was a reliable and valid instrument with an internal consistency reliability coefficient of. 80 was

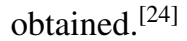

\subsection{Data analysis}

The collected data were organized, tabulated, and statistically analyzed using SPSS software (Statistical Package for the Social Sciences, version 26, SPSS Inc. Chicago, IL, USA). The categorical variables were represented as frequency and percentage. The continuous variables were represented as mean, and standard deviation. Mann-Whitney U test was used to compare the differences between two means of nonparametric variables. Whereas independent $t$ test as used to compare the differences between two means of parametric variables. The Chi-square test was conducted to compare the differences between categorical variables. Friedman's Test was conducted to compare repeated measures of non-parametric ordinal variables among the same subjects. Whereas repeated two-way ANOVA was conducted to compare repeated measures of parametric continuous variables. Statistically significant was considered as ( $p$-value $\leq .01 \&$ $.05)$.

\subsection{Ethical approval}

The study was approved by the Scientific Research Ethics Committee of Faculty of Nursing, Mansoura University (Resolution No:0223) and was conducted in accordance with the Declaration of Helsinki.

\section{RESUltS}

\subsection{Characteristics of the Participants}

A total of 74 BC adults' women participated in the study were allocated randomly into PMRT and control groups by simple random sampling, 37 in each arm. All participants completed all stages of the study from the beginning to the end without withdrawal. Table 1 showed that the study groups were well matched for education level, occupation, residence, and marital status. Thus, it is concluded that both groups were statistically homogeneous as regards sociodemographic variables. There were statistically significant differences between the PMRT, and control group related to their age, and the stage of disease $(p<.001)$. Most of them housewife $(83.8 \%$ \& $70.3 \%)$ in PMRT and control group, respectively. Most (78.4\%) of subjects were married. Majority of studied women in PMRT and control group (56.8\% \& 64.9\%) were in stage I \& II of illness, respectively. 


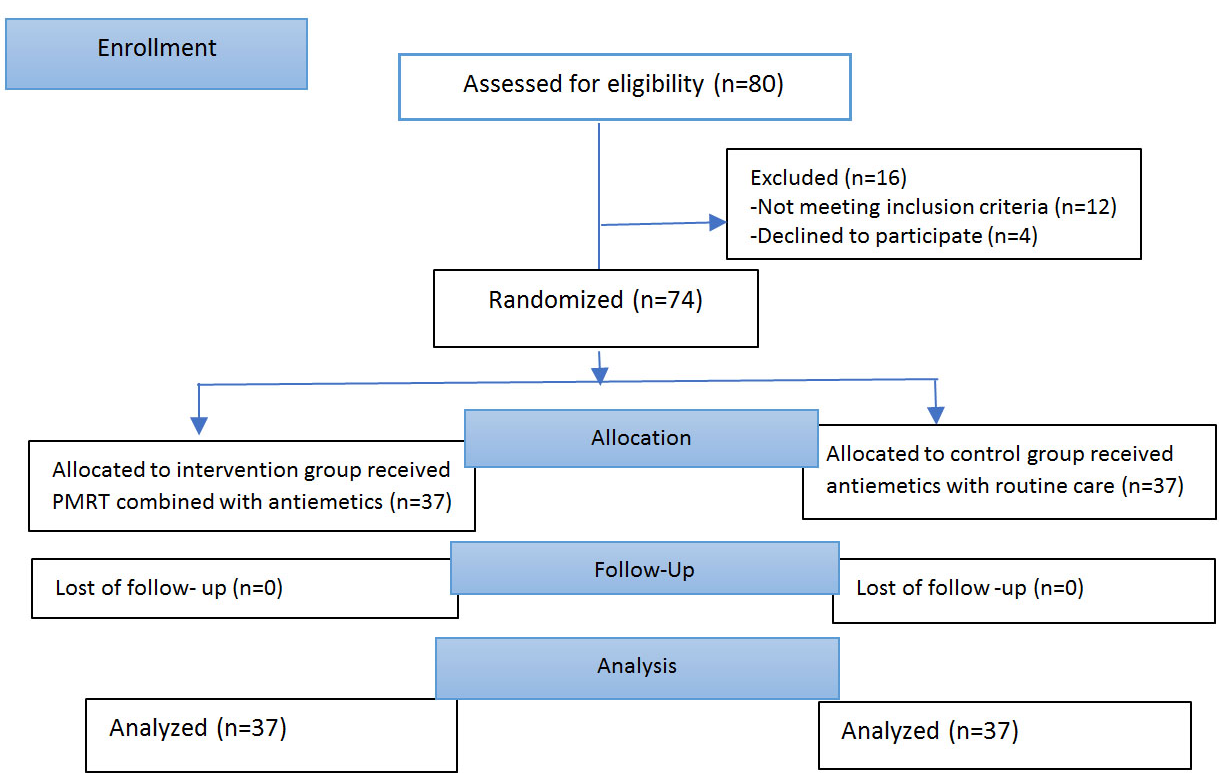

Figure 1. Flow-chart of study enrollment and participation

Table 1. Demographic characteristics and clinical data of the participants $(\mathrm{N}=74)$

\begin{tabular}{|c|c|c|c|c|c|}
\hline \multirow[t]{2}{*}{ Variables } & \multicolumn{2}{|c|}{$\begin{array}{l}\text { PMRT group } \\
(\mathbf{n}=\mathbf{3 7})\end{array}$} & \multicolumn{2}{|c|}{$\begin{array}{l}\text { Control group } \\
(n=37)\end{array}$} & \multirow[t]{2}{*}{$\chi^{2} / p_{1}$} \\
\hline & n & $\%$ & $\mathbf{n}$ & $\%$ & \\
\hline \multicolumn{6}{|l|}{ Age } \\
\hline $25-<35$ & 1 & 2.7 & 18 & 48.6 & \multirow{4}{*}{$\begin{array}{l}20.58 / \\
.000 * * \\
\mathrm{Z}=3.40 / \\
p_{2 .}=.001 * *\end{array}$} \\
\hline $35-<45$ & 17 & 45.9 & 8 & 21.6 & \\
\hline$\geq 45$ & 19 & 51.4 & 11 & 29.7 & \\
\hline Mean \pm SD & \multicolumn{2}{|c|}{$47.35 \pm 7.90$} & \multicolumn{2}{|c|}{$38.89 \pm 9.64$} & \\
\hline \multicolumn{6}{|l|}{ Education } \\
\hline Primary education & 5 & 13.5 & 5 & 13.5 & \multirow{3}{*}{$1.60 / .45$} \\
\hline Secondary education & 21 & 56.8 & 16 & 43.2 & \\
\hline university education & 11 & 29.7 & 16 & 43.2 & \\
\hline \multicolumn{6}{|l|}{ Residence } \\
\hline Urban & 7 & 18.9 & 10 & 27.0 & \multirow[t]{2}{*}{$0.69 / .41$} \\
\hline Rural & 30 & 81.1 & 27 & 73.0 & \\
\hline \multicolumn{6}{|l|}{ Occupation } \\
\hline Housewife & 31 & 83.8 & 26 & 70.3 & \multirow[t]{2}{*}{$1.91 / .18$} \\
\hline Employed & 6 & 16.2 & 11 & 29.7 & \\
\hline \multicolumn{6}{|l|}{ Marital status } \\
\hline Single & 3 & 8.1 & 3 & 8.1 & \multirow{4}{*}{$\begin{array}{l}0.000 / \\
1.00\end{array}$} \\
\hline Married & 29 & 78.4 & 29 & 78.4 & \\
\hline Widowed & 3 & 8.1 & 3 & 8.1 & \\
\hline Divorced & 2 & 5.4 & 2 & 5.4 & \\
\hline \multicolumn{6}{|l|}{ Stages of disease } \\
\hline Stage 1 & 0 & 0.0 & 24 & 64.9 & \multirow{4}{*}{$\begin{array}{l}41.68 / \\
.000 * *\end{array}$} \\
\hline Stage 2 & 21 & 56.8 & 9 & 24.3 & \\
\hline Stage 3 & 16 & 43.2 & 2 & 5.4 & \\
\hline Stage 4 & 0 & 0.0 & 2 & 5.4 & \\
\hline \multicolumn{6}{|c|}{$\begin{array}{l}\text { Note. } p<.05 / * * p<.01 ; p_{1} \text { : value of Chi-square test to illustrate statistically } \\
\text { significant changes between the PMRT and control group regarding categorical } \\
\text { personal characteristics. } p_{2} \text { : value of Mann-Whitney U test to illustrate statistically } \\
\text { significant changes between the PMRT and control group regarding continuous }\end{array}$} \\
\hline
\end{tabular}

Published by Sciedu Press

\subsection{Comparison of chemotherapy symptoms in PMRT and control group \\ 3.2.1 Effects of PMRT on frequency of nausea and vomit- ing of participants}

Table 2 illustrates that, there were highly statistically significant changes between the study groups related to frequency of vomiting and nausea at the four days of muscle relaxation training program where $(p<.001)$. The PMRT group experienced lower incidence of nausea and vomiting compared with control group particularly in the first four days after chemotherapy, in this time the findings were significant where $(p<.001)$. Nausea and vomiting were reported during the acute and late periods, while late symptoms were at their highest point on day 4 post chemotherapy and $\mathrm{BC}$ women in the control group were more likely to have delayed nausea and vomiting even after routine care. Interestingly, there were statistically significant changes in frequency of vomiting and nausea within the study groups post chemotherapy where $(p<.001)$.

\subsubsection{Effectiveness of PMRT on severity of vomiting and nausea of participants}

It is obvious from table 3 that there was a significant change in the severity of nausea- vomiting between the study groups, the PMRT group reporting lower severity when compared to the control group, the differences were particularly noticeable in the first four days post chemotherapy, at this point of time the findings were statistically significant $(p<.001)$. Additionally, there were statistically significant changes related to severity of vomiting and nausea within the PMRT and control group over the first four days of chemotherapy where $(p<.001)$. 
Table 2. Effects of PMRT on frequency of vomiting and nausea among BC women $(\mathrm{n}=74)$

\begin{tabular}{|c|c|c|c|c|c|c|c|c|c|c|c|}
\hline \multirow{2}{*}{$\begin{array}{l}\text { Frequency } \\
\text { of vomiting }\end{array}$} & \multicolumn{2}{|c|}{$\begin{array}{l}\text { PMRT group } \\
(\mathrm{n}=37)\end{array}$} & \multicolumn{2}{|c|}{$\begin{array}{l}\text { Control group } \\
(n=37)\end{array}$} & \multirow[t]{2}{*}{$\chi^{2} / p_{1}$} & \multirow{2}{*}{$\begin{array}{l}\text { Frequency } \\
\text { of nausea }\end{array}$} & \multicolumn{2}{|c|}{$\begin{array}{l}\text { PMRT group } \\
(\mathrm{n}=37)\end{array}$} & \multicolumn{2}{|c|}{$\begin{array}{l}\text { Control group } \\
(\mathrm{n}=37)\end{array}$} & \multirow[t]{2}{*}{$\chi^{2} / p_{1}$} \\
\hline & $\mathrm{n}$ & $\%$ & $\mathrm{n}$ & $\%$ & & & $\mathrm{n}$ & $\%$ & $\mathbf{n}$ & $\%$ & \\
\hline $1^{\text {st }}$ day & & & & & & $1^{\text {st }}$ day & & & & & \multirow{5}{*}{$\begin{array}{l}18.27 / \\
.000^{* *}\end{array}$} \\
\hline None & 12 & 32.4 & 0 & 0.0 & \multirow{4}{*}{$\begin{array}{l}20.00 / \\
.000 * *\end{array}$} & None & 8 & 21.6 & 0 & 0.0 & \\
\hline 1-2 times & 17 & 45.9 & 0 & 0.0 & & 1-2 times & 10 & 27.0 & 24 & 64.9 & \\
\hline 3-4 times & 8 & 21.6 & 24 & 64.9 & & 3-4 times & 10 & 27.0 & 11 & 29.7 & \\
\hline 5-6 times & 0 & 0.0 & 13 & 35.1 & & $5-6$ times & 9 & 24.3 & 2 & 5.4 & \\
\hline $2^{\text {nd }}$ day & & & & & & $2^{\text {nd }}$ day & & & & & \multirow{6}{*}{$\begin{array}{l}10.95 / \\
.000^{* *}\end{array}$} \\
\hline None & 22 & 59.5 & 0 & 0.0 & \multirow{5}{*}{$\begin{array}{l}40.34 / \\
.000^{* *}\end{array}$} & None & 16 & 43.2 & 4 & 10.8 & \\
\hline 1-2 times & 14 & 37.8 & 16 & 43.2 & & 1-2 times & 13 & 35.1 & 21 & 56.8 & \\
\hline 3-4 times & 1 & 2.7 & 18 & 48.6 & & 3-4 times & 7 & 18.9 & 8 & 21.6 & \\
\hline 5-6 times & 0 & 0.0 & 2 & 5.4 & & $5-6$ times & 1 & 2.7 & 4 & 10.8 & \\
\hline 7 and more & 0 & 0.0 & 1 & 2.7 & & 7 and more & 0 & 0.0 & 0 & 0.0 & \\
\hline $3^{\text {rd }}$ day & & & & & & $3^{\text {rd day }}$ & & & & & \multirow{5}{*}{$\begin{array}{l}18.95 / \\
.000^{* *}\end{array}$} \\
\hline None & 24 & 64.9 & 3 & 8.1 & \multirow{4}{*}{$\begin{array}{l}36.37 / \\
.000^{* *}\end{array}$} & None & 21 & 56.8 & 4 & 10.8 & \\
\hline 1-2 times & 13 & 35.1 & 14 & 37.8 & & 1-2 times & 14 & 37.8 & 25 & 67.5 & \\
\hline 3-4 times & 0 & 0.0 & 17 & 45.9 & & 3-4 times & 2 & 5.4 & 5 & 13.5 & \\
\hline $5-6$ times & 0 & 0.0 & 3 & 8.1 & & 5-6 times & 0 & 0.0 & 3 & 8.1 & \\
\hline $4^{\text {th }}$ day & & & & & & $4^{\text {th }}$ day & & & & & \\
\hline None & 24 & 64.9 & 4 & 10.8 & \multirow{4}{*}{$\begin{array}{l}33.32 / \\
.000 * *\end{array}$} & None & 21 & 56.8 & 4 & 10.8 & \multirow{4}{*}{$\begin{array}{l}18.95 / \\
.000^{* *}\end{array}$} \\
\hline 1-2 times & 13 & 35.1 & 14 & 37.8 & & 1-2 times & 14 & 37.8 & 25 & 67.6 & \\
\hline 3-4 times & 0 & 0.0 & 16 & 43.2 & & 3-4 times & 2 & 5.43 & 5 & 13.5 & \\
\hline 5-6 times & 0 & 0.0 & 3 & 8.1 & & 5-6 times & 0 & 0.0 & 3 & 8.1 & \\
\hline$\chi^{2} / p_{2}$ & \multicolumn{2}{|c|}{$\begin{array}{l}52.71 / \\
.000 * *\end{array}$} & \multicolumn{2}{|c|}{$\begin{array}{l}36.80 / \\
.000^{* *}\end{array}$} & & $\chi^{2} / p_{2}$ & \multicolumn{2}{|c|}{$\begin{array}{l}73.80 / \\
.000 * *\end{array}$} & \multicolumn{2}{|c|}{$\begin{array}{l}9.75 / \\
.02 *\end{array}$} & \\
\hline
\end{tabular}

Note. Progressive muscle relaxation training (PMRT); breast cancer (BC); ${ }^{*} p<.05 / * * p<.01 ; p_{1}$ : value of Chi-square test to illustrate statistically significant differences between PMRT and control group in each day./ $p_{2}$ : value of Friedman's Test to illustrate statistically significant differences between the four days within each group (PMRT and control group).

\subsection{Comparing duration of nausea and vomiting per hours over time in study groups}

When comparing duration of nausea between study groups, Table 4 clarified that, $(21.6 \%)$ of PMRT group experienced no nausea at all on the first day, reaching maximum (56.8\%) on 3rd day of chemotherapy. In the control group, (59.5\%) experienced nausea for 2-3 hours on the first day, reaching $(18.9 \%)$ at third day. There were statistically significant differences as regards duration of nausea in the two groups $(p<$ .001). Additionally, small amount of vomiting was reported by (64.9\%) of the PMRT group on day one after chemotherapy, declining steadily to reach $(35.1 \%)$ by day 3 . There were statistically significant differences in amount of vomiting in study groups $(p<.001)$. Overall, there were statistically significant changes between the PMRT and control group in amount of vomiting and duration of nausea at the first four days of chemotherapy where $(p<.001)$.

\subsubsection{Effects of PMRT on retching}

Table 5 revealed that, retching frequency and severity was statistically significant lower in the PMRT group compared to the control group across the four assessment days post chemotherapy $(p<.001)$. Furthermore, there were statistically significant changes in the frequency and severity of retching within the PMRT and control group after the first four days of chemotherapy where $(p<.001)$. Overall, its observed from table (1-5) that, severity, frequency, amount and duration of nausea, vomiting and retching was also significantly improved in the PMRT group than in the comparison group $(p<.001)$.

\subsubsection{Effects of PMRT on anxiety scores of participants}

It is evident from Table 6 that, there were no significant changes in the total anxiety scores between the two groups before chemotherapy administration $(p>.05)$. There was a trend toward a significant change in mean anxiety scores 
between PMRT, and control group after 7 and 14 days of chemotherapy $(p<.001)$. Interestingly, when analyzing the differences in anxiety score within PMRT group throughout the study period Table 6 illustrated that, there was statis- tically significant difference in mean anxiety score of the PMRT group before, after 7 days and at the end of 14 days of implementing muscle relaxation training program where $\mathrm{F}$ value $/ p$ value $=2,168.15 / .0000^{* * *}$.

Table 3. Comparing severity of vomiting and nausea over time in study groups $(n=74)$

\begin{tabular}{|c|c|c|c|c|c|c|c|c|c|c|c|}
\hline \multirow{2}{*}{$\begin{array}{l}\text { Severity of } \\
\text { vomiting }\end{array}$} & \multicolumn{2}{|c|}{$\begin{array}{l}\text { PMRT group } \\
(\mathbf{n}=\mathbf{3 7})\end{array}$} & \multicolumn{2}{|c|}{$\begin{array}{l}\text { Control group } \\
(\mathrm{n}=37)\end{array}$} & \multirow{2}{*}{$\chi^{2} / p_{1}$} & \multirow{2}{*}{$\begin{array}{l}\text { Severity } \\
\text { of nausea }\end{array}$} & \multicolumn{2}{|c|}{$\begin{array}{l}\text { PMRT group } \\
(\mathbf{n}=\mathbf{3 7})\end{array}$} & \multicolumn{2}{|c|}{$\begin{array}{l}\text { Control group } \\
(\mathrm{n}=37)\end{array}$} & \multirow[t]{2}{*}{$\chi^{2} / p_{1}$} \\
\hline & $\mathrm{n}$ & $\%$ & $\mathrm{n}$ & $\%$ & & & $\mathrm{n}$ & $\%$ & $n$ & $\%$ & \\
\hline \multicolumn{5}{|l|}{$1^{\text {st }}$ day } & \multirow{6}{*}{$\begin{array}{l}31.89 / \\
.000 * *\end{array}$} & $1^{\text {st }}$ day & & & & & \multirow{6}{*}{$\begin{array}{l}21.00 / \\
.000 * *\end{array}$} \\
\hline No & 11 & 29.7 & 0 & 0.0 & & No & 8 & 21.6 & 0 & 0.0 & \\
\hline Mild & 23 & 62.2 & 12 & 32.4 & & Mild & 20 & 54.1 & 13 & 35.1 & \\
\hline Moderate & 3 & 8.1 & 22 & 59.5 & & Moderate & 5 & 13.5 & 21 & 56.8 & \\
\hline Great & 0 & 0.0 & 3 & 8.1 & & Great & 4 & 10.8 & 2 & 5.4 & \\
\hline Severe & 0 & 0.0 & 0 & 0.0 & & Severe & 0 & 0.0 & 1 & 2.7 & \\
\hline \multicolumn{6}{|l|}{$2^{\text {nd }}$ day } & \multicolumn{5}{|l|}{$2^{\text {nd }}$ day } & \multirow{5}{*}{$\begin{array}{l}18.90 / \\
.000 * *\end{array}$} \\
\hline No & 21 & 56.8 & 3 & 8.1 & \multirow{4}{*}{$\begin{array}{l}31.50 / \\
.000 * *\end{array}$} & No & 16 & 43.2 & 3 & 8.1 & \\
\hline Mild & 16 & 43.2 & 16 & 43.2 & & Mild & 19 & 51.4 & 19 & 51.4 & \\
\hline Moderate & 0 & 0.0 & 15 & 40.5 & & Moderate & 2 & 5.4 & 14 & 37.8 & \\
\hline Great & 0 & 0.0 & 3 & 8.1 & & Great & 0 & 0.0 & 1 & 2.7 & \\
\hline \multicolumn{6}{|l|}{$3^{\text {rd }}$ day } & \multicolumn{6}{|l|}{$3^{\text {rd day }}$} \\
\hline No & 24 & 64.9 & 4 & 10.8 & \multirow{4}{*}{$\begin{array}{l}32.43 / \\
.000 * *\end{array}$} & No & 21 & 56.8 & 4 & 10.8 & \multirow{4}{*}{$\begin{array}{l}25.82 / \\
.000 * *\end{array}$} \\
\hline Mild & 13 & 35.1 & 15 & 40.5 & & Mild & 16 & 43.2 & 19 & 51.4 & \\
\hline Moderate & 0 & 0.0 & 17 & 45.9 & & Moderate & 0 & 0.0 & 14 & 37.8 & \\
\hline Great & 0 & 0.0 & 1 & 2.7 & & Great & 0 & 0.0 & 0 & 0.0 & \\
\hline \multicolumn{6}{|l|}{$4^{\text {th }}$ day } & \multicolumn{6}{|l|}{$4^{\text {th }}$ day } \\
\hline No & 24 & 64.9 & 4 & 10.8 & \multirow{4}{*}{$\begin{array}{l}32.43 / \\
.000 * *\end{array}$} & No & 21 & 56.8 & 4 & 10.8 & \multirow{4}{*}{$\begin{array}{l}25.82 / \\
.000 * *\end{array}$} \\
\hline Mild & 13 & 35.1 & 15 & 40.5 & & Mild & 16 & 43.2 & 19 & 51.4 & \\
\hline Moderate & 0 & 0.0 & 17 & 45.9 & & Moderate & 0 & 0.0 & 14 & 37.8 & \\
\hline Great & 0 & 0.0 & 1 & 2.7 & & Great & 0 & 0.0 & 0 & 0.0 & \\
\hline$\chi^{2} / p_{2}$ & \multicolumn{2}{|c|}{$\begin{array}{l}39.37 / \\
.000^{* *}\end{array}$} & \multicolumn{3}{|c|}{$\begin{array}{l}20.11 / \\
.000^{* *}\end{array}$} & $\chi^{2} / p_{2}$ & \multicolumn{2}{|c|}{$\begin{array}{l}54.85 / \\
.000^{* *}\end{array}$} & \multicolumn{2}{|c|}{$\begin{array}{l}27.97 / \\
.000^{* *}\end{array}$} & \\
\hline
\end{tabular}

Note. Progressive muscle relaxation training (PMRT); ${ }^{*} p<.05 / * * p<.01 ; p_{1}$ : value of Chi-square test to illustrate statistically significant changes between the PMRT and control group in each day/p2: value of Friedman's Test to illustrate statistically significant differences between the four days within each group (PMRT and control group).

\section{Discussion}

The present study aimed to evaluate the efficacy of PMRT exercise on CINV and anxiety among breast cancer women. The results confirm the hypothesis (1) that, "The PMRT group would show significantly greater improvements in severity, frequency, and duration, of chemotherapyassociated nausea-vomiting and retching than the control group participants immediately and for a week postchemotherapy." The women participating in PMRT, and control group included in the study were identical for sociodemographic data such as education level, occupation, marital status, and residence. There were statistically significant changes between PMRT, and control group related to their age, and the stage of disease. In this study, more than half of the participants in the PMRT group and one third of the control group were past their fourth decade.

Similar study pointed out that, more than one third of the individuals in the intervention and the control group were close to the fifth decade of life. ${ }^{[25]}$ In the present study, majority of the patients were having stage II BC in the PMRT group. This comes in line with a study by Bhatia and associates who reported that majority of women in experimental group were having stage II BC. ${ }^{[25]}$ 
Table 4. Comparison of nausea and vomiting (amount \& duration) between the study groups $(\mathrm{n}=74)$

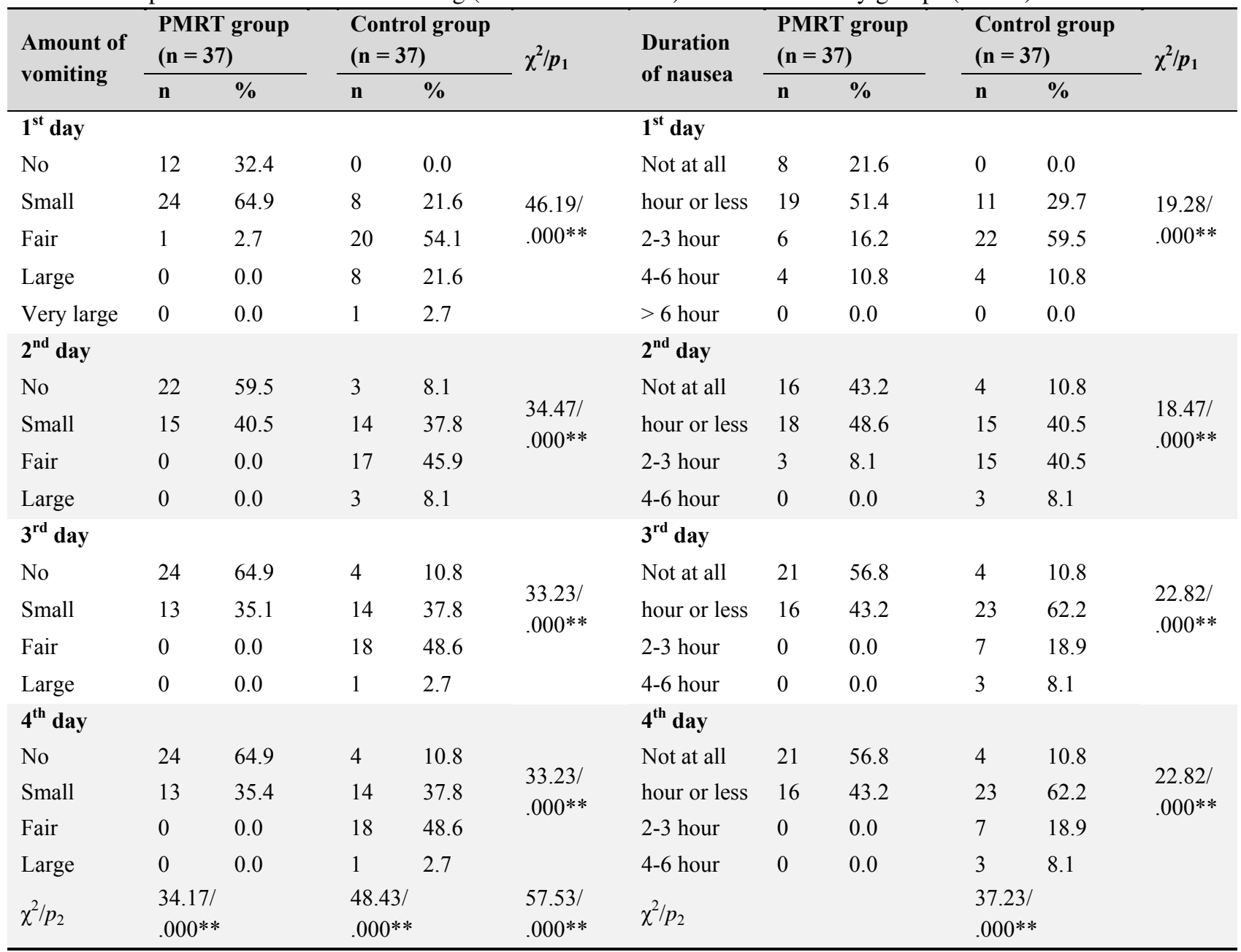

Note. Progressive muscle relaxation training (PMRT); ${ }^{*} p<.05 / * * p<.01 ; p_{1}$ : value of Chi-square test to illustrate statistically significant changes between the PMRT and control group in each day $/ p_{2}$ : value of Friedman's Test to illustrate statistically significant differences between the four days within each group (PMRT and control group).

The results of the current study proved that participants who received PMRT in conjunction with antiemetics experienced more improvement in post chemotherapy vomiting and nausea as shown by frequency, severity, and duration. Interestingly have better control over early and late vomiting and nausea compared to control group who received antiemetic and routine care. The changes between the control and PMRT group were considerably evident especially at the first four days.

In repeated measurements, PMRT group showed highly statistically significant decreases in frequency, severity, duration and amount of nausea-vomiting, and retching starting from the second day, where the changes became smaller between the two groups from the 3rd day of exercise, this results recommends that, the effectiveness of PMRT on nauseavomiting and retching were most evident in first week of receiving chemotherapeutic agents when the $\mathrm{BC}$ women are unfamiliar with a chemotherapy's nature and distressing symptoms. At this time, cancer patients urgently need effective mechanisms to counteract the painful symptoms of chemotherapy. From all the previous results, we conclude that, there were highly statistically significant differences between PMRT and control group related frequency, severity, and amount of vomiting- nausea, and retching at the four days of muscle relaxation training program .

These results are consistent with several studies in different countries. ${ }^{[18,26]}$ In randomized controlled trials (RCTs), the PMRT was dramatically reduced the frequency, severity, duration of nausea-vomiting and retching. ${ }^{[13]}$ Similarly, in the RCT, the results revealed that the gradual relaxation of the muscles caused changes in vomiting, nausea and physiological state, and these changes were statistically significant. In the present study, PMRT sessions were started at the first chemotherapeutic dose, which had a good effect in decreasing nausea-vomiting and retching. ${ }^{[27]}$ 
Table 5. Comparison of changes in retching between the PMRT and control group $(\mathrm{n}=74)$

\begin{tabular}{|c|c|c|c|c|c|c|c|c|c|c|c|}
\hline \multirow{2}{*}{$\begin{array}{l}\text { Frequency } \\
\text { of retching }\end{array}$} & \multicolumn{2}{|c|}{$\begin{array}{l}\text { PMRT group } \\
(\mathrm{n}=37)\end{array}$} & \multicolumn{2}{|c|}{$\begin{array}{l}\text { Control group } \\
(n=37)\end{array}$} & \multirow[t]{2}{*}{$\chi^{2} / p_{1}$} & \multirow{2}{*}{$\begin{array}{l}\text { Severity } \\
\text { of } \\
\text { retching }\end{array}$} & \multicolumn{2}{|c|}{$\begin{array}{l}\text { PMRT group } \\
(\mathrm{n}=37)\end{array}$} & \multicolumn{2}{|c|}{$\begin{array}{l}\text { Control group } \\
(n=37)\end{array}$} & \multirow[t]{2}{*}{$\chi^{2} / p_{2}$} \\
\hline & $n$ & $\%$ & $\mathrm{n}$ & $\%$ & & & $n$ & $\%$ & $n$ & $\%$ & \\
\hline $1^{\text {st }}$ day & & & & & \multirow{6}{*}{$\begin{array}{l}39.24 / \\
.000 * *\end{array}$} & $\mathbf{1}^{\text {st }}$ day & & & & & \multirow{6}{*}{$\begin{array}{l}32.52 / \\
.000 * *\end{array}$} \\
\hline None & 8 & 21.6 & 0 & 0.0 & & No & 8 & 21.6 & 0 & 0.0 & \\
\hline 1-2 times & 26 & 70.3 & 8 & 21.6 & & Mild & 26 & 70.3 & 11 & 29.7 & \\
\hline 3-4 times & 3 & 8.1 & 16 & 43.2 & & Moderate & 3 & 8.1 & 22 & 59.5 & \\
\hline 5-6 times & 0 & 0.0 & 10 & 27.0 & & Great & 0 & 0.0 & 4 & 10.8 & \\
\hline 7 and more & 0 & 0.0 & 3 & 8.1 & & Severe & 0 & 0.0 & 0 & 0.0 & \\
\hline \multicolumn{6}{|l|}{$2^{\text {nd }}$ day } & \multicolumn{5}{|l|}{$2^{\text {nd }}$ day } & \multirow{5}{*}{$\begin{array}{l}32.52 / \\
.000 * *\end{array}$} \\
\hline None & 20 & 54.1 & 3 & 8.1 & \multirow{4}{*}{$\begin{array}{l}32.86 / \\
.000 * *\end{array}$} & No & 20 & 54.1 & 4 & 10.8 & \\
\hline 1-2 times & 17 & 45.9 & 14 & 37.8 & & Mild & 17 & 45.9 & 12 & 32.4 & \\
\hline 3-4 times & 0 & 0.0 & 12 & 32.4 & & Moderate & 0 & 0.0 & 18 & 48.6 & \\
\hline 5-6 times & 0 & 0.0 & 8 & 21.6 & & Great & 0 & 0.0 & 3 & 8.1 & \\
\hline \multicolumn{6}{|l|}{$3^{\text {rd }}$ day } & \multicolumn{6}{|l|}{$3^{\text {rd }}$ day } \\
\hline None & 25 & 67.6 & 4 & 10.8 & \multirow{4}{*}{$\begin{array}{l}30.10 / \\
.000 * *\end{array}$} & No & 23 & 62.2 & 4 & 10.8 & \multirow{4}{*}{$\begin{array}{l}23.70 / \\
.000 * *\end{array}$} \\
\hline 1-2 times & 10 & 27.0 & 14 & 37.8 & & Mild & 12 & 32.4 & 20 & 54.1 & \\
\hline 3-4 times & 2 & 5.4 & 11 & 29.7 & & Moderate & 2 & 5.4 & 10 & 27.0 & \\
\hline 5-6 times & 0 & 0.0 & 8 & 21.6 & & Great & 0 & 0.0 & 3 & 8.1 & \\
\hline \multicolumn{6}{|l|}{$4^{\text {th }}$ day } & \multicolumn{6}{|l|}{$4^{\text {th }}$ day } \\
\hline None & 25 & 67.6 & 4 & 10.8 & \multirow{4}{*}{$\begin{array}{l}30.10 / \\
.000 * *\end{array}$} & No & 23 & 62.2 & 4 & 10.8 & \multirow{4}{*}{$\begin{array}{l}23.70 / \\
.000 * *\end{array}$} \\
\hline 1-2 times & 10 & 27.0 & 14 & 37.8 & & Mild & 12 & 32.4 & 20 & 54.1 & \\
\hline 3-4 times & 2 & 5.4 & 11 & 29.7 & & Moderate & 2 & 5.4 & 10 & 27.0 & \\
\hline 5-6 times & 0 & 0.0 & 8 & 21.6 & & Great & 0 & 0.0 & 3 & 8.1 & \\
\hline$\chi^{2} / p_{2}$ & \multicolumn{2}{|c|}{$\begin{array}{l}41.12 / \\
.000^{* *}\end{array}$} & \multicolumn{3}{|c|}{$\begin{array}{l}34.65 / \\
.000 * *\end{array}$} & $\chi^{2} / p_{2}$ & \multicolumn{2}{|c|}{$\begin{array}{l}34.11 / \\
.000^{* *}\end{array}$} & \multicolumn{2}{|c|}{$\begin{array}{l}30.55 / \\
.000^{* *}\end{array}$} & \\
\hline
\end{tabular}

Note. Progressive muscle relaxation training (PMRT); ${ }^{*} p<.05 /{ }^{* *} p<.01 ; p_{1}$ : value of Chi-square test to illustrate statistically significant differences between the study group and control group in each day $/ p_{2}$ : value of Friedman's Test to illustrate statistically significant differences between the four days within each group (PMRT and control group).

Table 6. Effects of PMRT on anxiety scores of participants $(\mathrm{n}=74)$

\begin{tabular}{llll}
\hline $\begin{array}{l}\text { Total anxiety } \\
\text { score }\end{array}$ & $\begin{array}{l}\text { PMRT group } \\
(\mathbf{n}=\text { 37) }\end{array}$ & $\begin{array}{l}\text { Control group } \\
(\mathbf{n}=37)\end{array}$ & $\begin{array}{l}\boldsymbol{t} \text {-value/ } \\
\boldsymbol{n} \text { v value }\end{array}$ \\
\cline { 2 - 3 } & Mean \pm SD & Mean \pm SD & \\
\hline Before & $42.13 \pm 1.91$ & $42.08 \pm 2.19$ & $0.11 / 0.91$ \\
After 7 days & $17.91 \pm 2.24$ & $42.14 \pm 1.91$ & $49.95 / .000^{* *}$ \\
After 17days & $17.91 \pm 2.24$ & $42.14 \pm 1.91$ & $49.95 / .000^{* *}$ \\
F value $/ p$ value & $2168.15 / .0000^{* *}$ & $0.11 / .74$ & \\
\hline
\end{tabular}

Note. Progressive muscle relaxation training (PMRT); ${ }^{*} p<.05 /{ }^{* *} p<.01 ; p 1$ : value of independent $t$ test illustrates statistically significant changes between the PMRT and control group in each phase of training program. / p2: value of repeated two-way ANOVA to illustrate statistically significant differences between three phases of training program for each group (PMRT and control group)

It is also worth noting, these results supported other studies reporting that nurses can train oncology patients to practice PMRT, as it can be easily learned, and patients can practice on their own. ${ }^{[28]}$ Previous results confirmed the efficacy of using PMRT as a non-pharmacological drug for decreasing the side effects of chemotherapy. Additionally, the previ- ous finding is proportionate with a study confirmed that, PMRT, improved anticipatory, early, and late CINV in conjunction with antiemetics than using pharmacological treatment alone. ${ }^{[29,30]}$ Therefore, PMRT is an effective method with antiemetics administered to $\mathrm{BC}$ patients to decrease the severity, duration of vomiting and nausea. ${ }^{[27]}$

When considering the effectiveness of PMRT on reducing anxiety between the study groups, according to Rhodes Index scores, the results of this study indicated that, BC women of PMRT group illustrated significant decrease in its mean anxiety scores after two weeks, when compared to mean anxiety scores in comparable group. There were statistically significant differences between PMRT and control group in their anxiety level post one and two week of chemotherapy. Additionally, there was a trend toward a significant difference in anxiety level within PMRT group after two weeks of implementing PMRT.

Overall, the findings of the present study indicated that, the anxiety related to $\mathrm{NV}$ and retching experienced by 
BC women after practicing of PMRT sessions was lower than that of the control group with a statistically significant changes. This may be due to meticulous attention and care provided by researcher during the program implementation. These findings support our 2nd hypothesis, proposed that "Women with breast cancer who receive PMRT will have significantly less anxiety than women in the control group after two weeks of chemotherapy".

This result is congruent with RCT on preventing anxiety and depression in cancer patients by applying PMRT sessions. The experimental group significantly decreases total hospital anxiety and depression score. ${ }^{[31]}$ Which has been confirmed by other study proved that PMRT had a good effect in reducing anxiety and depression among patients. ${ }^{[32]}$ A study conducted in South Korea pointed out that PMRT reduced the adverse effects of chemotherapy in women with breast cancer. $^{[33]}$

In this regard, similar results were seen in the study conducted by Kaur and others which was a randomized controlled trail on preventing anxiety, depression, and fatigue among patients with cervical cancer, the intervention significantly reduces total hospital anxiety and depression score. This decrease was observed in both subscales of anxiety and depression. ${ }^{[26]}$ In this context, two recent studies conclude that there is a positive effect of PMRT in reducing anxiety in breast cancer patients. ${ }^{[1,34]}$

\section{Conclusion}

In conclusion, the current study highlighted sufficient evidence that PMRT combined with antiemetic drugs were effective in decreasing the severity, frequency, and duration of CINV, retching, and anxiety among BC patients. The results also pointed out, the possibility to safely integrate PMRT with antiemetics into clinical practice, to control such painful side effects of chemotherapy.

\section{Relevance to clinical practice}

According to the study findings, (1) the hospital should provide instructions and demonstration videos that include the PMRT technique to be used by the patient in chemotherapy department, (2) PMRT should be demonstrated using different measurement scales in all cancer stage, (3) conduct larger, longer follow-up studies in groups of patients receiving different chemotherapy protocols to obtain a higher probability outcome.

\section{FUNDING}

The authors did not receive any financial support to conduct or publish this research article.

\section{CONFlicts of InTERest Disclosure}

The authors declare that they have no conflict of interest.

\section{REFERENCES}

[1] Yudono D. The Effect of Progressive Muscle Relaxation Therapy on Anxiety of Patients with Chemotherapy Measures at Dadi Keluarga Hospital Banyumas. Advances in Health Sciences Research. 2019; 20: $54-58$.

[2] Ibrahim AS, Khaled HM, Mikhail NN, et al. Cancer incidence in Egypt: Results of the national population-based cancer registry program. J Cancer Epidemiol. 2014; 437971. PMid:25328522 https://doi.org/10.1155/2014/437971

[3] Charalambous A, Giannakopoulou M, Bozas E, et al. Guided imagery, and progressive muscle relaxation as a cluster of symptoms management intervention in patients receiving chemotherapy: a randomized control trial. PloS One. 2016; Jun 24; 11(6): e0156911. PMid:27341675 https://doi.org/10.1371/journal . pone.0 156911

[4] DeVita, Hellman, Rosenberg. Cancer principles and practice of oncology: Side effects of cancer. 8th ed. Philadelphia (USA): Williams and wilkins; 2008; 2639-41.

[5] Shakiba M, Parsi H, Pahlavani Shikhi Z, et al. The Effect of PsychoEducation Intervention Based on Relaxation Methods and Guided Imagery on Nausea and Vomiting of Pregnant Women. J Fam Reprod Health 2019; 13(1): 47-55. https://doi.org/10.18502/jfrh. v13i1. 1612

[6] Dikmen HA, Terzioglu F. Effects of reflexology and progressive muscle relaxation on pain, fatigue, and quality of life during chemotherapy in gynecologic cancer patients. Pain Management Nursing. 2019; 20(1): 47-5. PMid:29776873 https://doi.org/10.1016/j.pm n. 2018.03.001

[7] Steinberg BA, Klatt M, Duchemin AM. Feasibility of a mindfulnessbased intervention for surgical intensive care Unit personnel. Am. J. Crit. Care. 2017; 26(1): 10-18. PMid:27965223 https ://doi .or g/10.4037/ajcc2017444

[8] Troxel WM, Buysse DJ, Matthews KA, et al. Sleep symptoms predict the development of the metabolic syndrome. Sleep. 2010; 33(12): 1633-1640. PMid:21120125 https://doi.org/10.1093/sleep/33.12.1633

[9] Nasiri S, Akbari H, Tagharrobi L, et al. The effect of progressive muscle relaxation and guided imagery on stress, anxiety, and depression of pregnant women referred to health centers. J Educ Health Promot. 2018; 7: 41.

[10] Tian X, Xu LL, Tang RY, et al. Role of progressive muscle relaxation in preventing and alleviating of nausea and vomiting caused by chemotherapy among cancer patients: a protocol of systematic review. TMR Integrative Nursing. 2019; 3(6): 226-230. https: //doi.org/10.12032/tmrin20191213

[11] Sahin ZA, Dayapog N. Effect of progressive relaxation exercises on fatigue and sleep quality in patients with chronic obstructive lung 
disease (COPD). Complement. Ther. Clin. Practice. 2015; 21(4): 277281. PMid:26573455 https://doi.org/10.1016/j.ctcp. 201 5.10 .002

[12] Borison HL, McCarthy LE. Neuropharmacologic mechanisms of emesis. In: Laszlo J (ed) Antiemetics and cancer chemotherapy. Williams \& Wilkins, Baltimore. 1983; 6-20.

[13] Anugrahini H, Nuswantoro D, Sriyono. Progressive Muscle Relaxation and Symptoms of Women with Breast Cancer Undergoing Adjuvant chemotherapy. Indian Journal of Forensic Medicine \& Toxicology. 2019. https://doi .org/10.5958/0973-9130.2019.0 0535.8

[14] Nabila ES. Nursing Intervention Based on Neuman's Model for Chemotherapy-Induced Nausea and Vomiting among Women with Breast Cancer. IOSR Journal of Nursing and Health Science (IOSRJNHS). 2019; 8(2): 75-90

[15] Fessele KS. Managing the multiple causes of nausea and vomiting in the patient with cancer. Oncology Nursing Forum. 1996; 23(9): e1409-e1415.

[16] Turini M, Piovesana V, Ruffo P. An assessment of chemotherapy-induced nausea and vomiting direct costs in three EU countries. Drugs Context. 2015; 4: 212-285. PMid:26322114 https://doi. org/10.7573/dic. 212285

[17] ArakawaS. Relaxation to reduce nausea, vomiting, and anxiety induced by chemotherapy in Japanese patients. Cancer Nursing. 1997; 20(5): 342e349. PMid:9394056 https : //doi .org/10.1097/00 002820-199710000-00005

[18] Molassiotis HP, Yung BM, Yam FY, et al. The effectiveness of progressive muscle relaxation training in managing chemotherapyinduced nausea and vomiting in Chinese breast cancer patients: a randomised controlled trial, Support. Care Cancer. 2002; 10(3): 237-46. PMid:11904789 https ://doi .org/10.1007/s00520-001-032 9-9

[19] Snyder M, Lindquist R. Complementary/Alternative Therapies in Nursing. 4th ed. Springer Publishing Company, New York: 2002.

[20] Chan CWH, Richardson A, Richardson J. Evaluating complex intervention: a process evaluation of a psychoeducational intervention in patients receiving palliative lung cancer radiotherapy. Contemporary Nurse. 2012; 40(2): 234-244. PMid:22554216 https: //doi.org/10.5172/conu.2012.40.2.234

[21] RhodesV A, McDanielR W. The Index of Nausea, Vomiting, and Retching: a new format of the lndex of Nausea and Vomiting. In Oncology Nursing Forum. 1999; 26(5): 889-894.

[22] Kim TH, Choi BM, Chin JH, et al. The reliability and validity of the Rhodes index of nausea, vomiting and retching in postoperative nausea and vomiting. Korean J Anesthesiol. 2007; 52(6): S59-S65. https://doi.org/10.4097/kjae.2007.52.6.S59

[23] Zung WW. How normal is Anxiety? (CurrentConcepts)/WWÊ.Zung. Upjohn Company. 1980.
[24] Ramirez SZ, Lukenbill J. Psychometric properties of the Zung SelfRating Anxiety Scale for adults with intellectual disabilities (SASID). Journal of Developmental and Physical Disabilities. 2008; 20(6): 573-580. https://doi.org/10.1007/s10882-008-9120-x

[25] Bhatia M, Verma M, Kumar R. Effectiveness of progressive muscle relaxation therapy on physical symptoms among cancer patients receiving chemotherapy admitted in Cancer Unit of Institute of Liver and Biliary Sciences, Delhi. Int J Sci \& Healthcare Res. 2020; 5(4): 221-232.

[26] Kurt B, Kapucu S.The effect of relaxation exercises on symptom severity in patients with breast cancer undergoing adjuvant chemotherapy: An open label non-randomized controlled clinical trial European Journal of Integrative Medicine. 2018; 22(2018): 54 61. https://doi.org/10.1016/j. eujim. 2018.08.002

[27] Carvalho EC, Martins FTM, Santos CB. A pilot study of a relaxation technique for management of nausea and vomiting in patients receiving cancer chemotherapy. Cancer Nurs. 2007; 30(2): 163-167. PMid:17413783 https://doi.org/10.1097/01. NCC.0000265 $007.87311 . \mathrm{d} 0$

[28] Molassiotis A, Yung H, Yam B. The clinical management of chemotherapy-induced nausea and vomiting with adjuvant progressive muscle relaxation training and imagery techniques in breast cancer patients. Hong Kong Med. 2006.

[29] Amer R, El-SayedR, Hamad M. The Effect of Applying a Progressive Muscle Relaxation Technique on Nausea and Vomiting Induced by Chemotherapy among Leukemic Children. American Journal of Nursing Research. 2020; 8(3): 331-343.

[30] Mustian KM, Devine K, Ryan JL, et al. Treatment of nausea and vomiting during chemotherapy. US Oncology \& Hematology. 2011; 7(2): 91.

[31] Kaur M, Agnihotri M, Das K, et al. Effectiveness of an Interventional Package on the Level of Anxiety, Depression, and Fatigue among Patients with Cervical Cancer. Asia Pac J Oncol Nurs. 2018; 5: 195200. PMid:29607380 https://doi.org/10.4103/apjon.apjo n_56_17

[32] Herizchi S, Asvadi I, Piri I, et al. Efficacy of Progressive Muscle Relaxation Training on Anxiety, Depression and Quality of Life in Cancer Patients Undergoing Chemotherapy at Tabriz Hematology and Oncology Research Center, Iran in 2010 Middle East. Journal of Cancer. 2012; 3 (1): 9-13.

[33] Yoo HJ, Ahn SH, Kim SB, et al. Efficacy of progressive muscle relaxation training and guided imagery in reducing chemotherapy side effects in patients with breast cancer and in improving their quality of life. Support Care Cancer. 2005; 13: 826-33. PMid:15856335 https://doi.org/10.1007/s00520-005-0806-7

[34] Ozgundondu B, Gok Metin Z. Effects of progressive muscle relaxation combined with music on stress, fatigue, and coping styles among intensive care nurses Intensive \& Critical Care Nursing. 2019; 54-63. PMid:31371164 https://doi.org/10.1016/j.iccn.2 019.07 .007 